\title{
Necessary and Sufficient Conditions for Positive Solutions of Second-Order Nonlinear Dynamic Equations on Time Scales
}

\author{
Zhi-Qiang Zhu \\ Department of Computer Science, Guangdong Polytechnic Normal University, Guangzhou 510665, China \\ Correspondence should be addressed to Zhi-Qiang Zhu, z3825@163.com
}

Received 28 December 2010; Revised 22 March 2011; Accepted 13 April 2011

Academic Editor: Mihai Putinar

Copyright (C) 2011 Zhi-Qiang Zhu. This is an open access article distributed under the Creative Commons Attribution License, which permits unrestricted use, distribution, and reproduction in any medium, provided the original work is properly cited.

This paper is concerned with the existence of nonoscillatory solutions for the nonlinear dynamic equation $\left(p(t)(\psi \circ x)\left(x^{\sigma}\right)^{\Delta}\right)^{\Delta}+q(t) f \circ x^{\sigma}=0$ on time scales. By making use of the generalized Riccati transformation technique, we establish some necessary and sufficient criteria to guarantee the existence. The last examples show that our results can be applied on the differential equations, the difference equations, and the $q$-difference equations.

\section{Introduction}

In the recent decade there have been many literatures to study the oscillatory properties for second-order dynamic equations on time scales; see, for example, [1-11] and the references therein. In particular, the dynamic equation of the form

$$
\left(p(t) x^{\Delta}\right)^{\Delta}+q(t) f \circ x^{\sigma}=0
$$

has been attracting one's interesting; see, for example, $[3,5,8]$. Motivated by the papers mentioned as above, in this paper we consider the existence of nonoscillatory solutions for nonlinear dynamic equation

$$
\left(p(t)(\psi \circ x)\left(x^{\sigma}\right)^{\Delta}\right)^{\Delta}+q(t) f \circ x^{\sigma}=0
$$

on a time scale $\mathbb{T}$, where $x^{\sigma}=x \circ \sigma$. 
Referring to $[12,13]$, a time scale $\mathbb{T}$ can be defined as an arbitrary nonempty subset of the set $\mathbb{R}$ of real numbers, with the properties that every Cauchy sequence in $\mathbb{T}$ converges to a point of $\mathbb{T}$ with the possible exception of Cauchy sequences which converge to a finite infimum or finite supremum of $\mathbb{T}$. On any time scale $\mathbb{T}$, the forward and backward jump operators are defined, respectively, by

$$
\sigma(t):=\inf \{s \in \mathbb{T}: s>t\}, \quad \rho(t):=\sup \{s \in \mathbb{T}: s<t\},
$$

where $\inf \emptyset:=\sup \mathbb{T}$ and $\sup \emptyset:=\inf \mathbb{T}$. A point $t \in \mathbb{T}$ is said to be right-scattered if $\sigma(t)>t$, right-dense if $\sigma(t)=t$, left-scattered if $\rho(t)<t$, and left-dense if $\rho(t)=t$. A derived set from $\mathbb{T}$ is defined as follows: $\mathbb{T}^{k}=\mathbb{T}-\{m\}$ when $\mathbb{T}$ has a left-scattered maximum $m$, otherwise $\mathbb{T}^{k}=\mathbb{T}$.

Definition 1.1. For a function $f: \mathbb{T} \rightarrow \mathbb{R}$ and $t \in \mathbb{T}^{k}$, we define the delta-derivative $f^{\Delta}(t)$ of $f(t)$ to be the number (provided it exists) with the property that, for any $\varepsilon>0$, there is a neighborhood $U$ of $t$ (i.e., $U=(t-\delta, t+\delta) \cap \mathbb{T}$ for some $\delta$ ) such that

$$
\left|[f(\sigma(t))-f(s)]-f^{\Delta}(t)[\sigma(t)-s]\right| \leq \varepsilon|\sigma(t)-s| \quad \forall s \in U
$$

We say that $f$ is delta-differentiable (or in short: differentiable) on $\mathbb{T}^{k}$ provided $f^{\Delta}(t)$ exists for all $t \in \mathbb{T}^{k}$.

For two differentiable functions $f$ and $g$ at $t \in \mathbb{T}^{k}$ with $g(t) g(\sigma(t)) \neq 0$, it holds that

$$
\begin{gathered}
(f g)^{\Delta}(t)=f^{\Delta}(t) g(t)+f(\sigma(t)) g^{\Delta}(t) \\
\left(\frac{f}{g}\right)^{\Delta}(t)=\frac{f^{\Delta}(t) g(\sigma(t))-f(\sigma(t)) g^{\Delta}(t)}{g(t) g(\sigma(t))} .
\end{gathered}
$$

Definition 1.2. A function $F: \mathbb{T} \rightarrow \mathbb{R}$ is called an antiderivative of $f$ provided $F^{\Delta}(t)=f(t)$ holds for all $t \in \mathbb{T}^{k}$. By the antiderivative, the Cauchy integral of $f$ is defined as $\int_{a}^{b} f(s) \Delta s=$ $F(b)-F(a)$, and $\int_{a}^{\infty} f(s) \Delta s=\lim _{t \rightarrow \infty} \int_{a}^{t} f(s) \Delta s$.

Definition 1.3. Let $f: \mathbb{T} \rightarrow \mathbb{R}$ be a function, where $f$ is called right-dense continuous (rdcontinuous) if it is right continuous at right-dense points in $\mathbb{T}$ and its left-sided limits exist (finite) at left-dense points in $\mathbb{T}$.

To distinguish from the traditional interval in $\mathbb{R}$, we define the interval in $\mathbb{T}$ by

$$
[a, \infty)_{\mathbb{T}}:=\{t \in \mathbb{T}: a \leq t<\infty\}
$$

Let $C_{\mathrm{rd}}(\mathbb{T})$ (or $C_{\mathrm{rd}}(\mathbb{T}, \mathbb{R})$ ) denote the set of all rd-continuous functions defined on $\mathbb{T}$, and $C_{\mathrm{rd}}^{1}(\mathbb{T})\left(\right.$ or $\left.C_{\mathrm{rd}}^{1}(\mathbb{T}, \mathbb{R})\right)$ denote the set of all differentiable functions whose derivative is rdcontinuous.

Since we are interested in the existence of nonoscillatory solutions of (1.2), we make the blanket assumption that $\inf \mathbb{T}=t_{0}$ and $\sup \mathbb{T}=\infty$. As defined in [1], by a solution of (1.2) 
we mean a nontrivial real function $x(t)$, with $x(t) \in C_{\mathrm{rd}}^{1}\left[t_{1}, \infty\right)_{\mathbb{T}}$ and $p(t) \psi(x(t))\left(x^{\sigma}(t)\right)^{\Delta} \in$ $C_{\text {rd }}^{1}\left[t_{1}, \infty\right)_{\mathbb{T}}$ for some $t_{1} \geq t_{0}$, which satisfies $(1.2)$ on $\left[t_{1}, \infty\right)_{\mathbb{T}}$. A solution of (1.2) is said to be nonoscillatory if it is eventually positive or eventually negative.

\section{Preliminaries}

Let us assume in (1.2) under consideration that

(H1) $\sigma \in C_{\text {rd }}^{1}(\mathbb{T}, \mathbb{T})$ with $\sigma^{\Delta}(t)>0$ on $\mathbb{T}$,

$(\mathrm{H} 2) p, q \in C_{\mathrm{rd}}(\mathbb{T}, \mathbb{R})$ and there exists a $t_{1} \in \mathbb{T}$ such that $p(t)>0$ and $q(t) \geq 0$ on $\left[t_{1}, \infty\right)_{\mathbb{T}}$ and $\int_{t_{1}}^{\infty}(\Delta s / p(s))=\infty, \int_{t_{1}}^{\infty} q(s) \Delta s<\infty$; also, $q$ is not identically zero on $\left[t_{1}, \infty\right)_{\mathbb{T}}$,

(H3) $\psi \in C^{1}\left(\mathbb{R}, \mathbb{R}^{+}\right)$with $\psi(x) \leq m_{0}$ on $\mathbb{R}$ for some constant $m_{0}>0$ and, $\psi$ is nonincreasing on $[0, \infty)$ and nondecreasing on $(-\infty, 0]$,

(H4) $f \in C^{1}(\mathbb{R}, \mathbb{R})$ satisfies that $x f(x)>0$ for $x \neq 0, f^{\prime}(x) \geq m_{1}$ on $\mathbb{R}$ for some constant $m_{1}>0$, and $f^{\prime}$ is nondecreasing on $[0, \infty)$ and nonincreasing on $(-\infty, 0]$.

Suppose that $\tilde{\mathbb{T}}=\left\{x(t) \in C_{\mathrm{rd}}(\mathbb{T}, \mathbb{R}): t \in \mathbb{T}\right\}$ is a time scale. By $\widetilde{\sigma}$ and $\tilde{\rho}$ we denote the forward and backward jump operators on $\tilde{\mathbb{T}}$, respectively, and by $\widetilde{\Delta}$ we denote the derivative on $\widetilde{\mathbb{T}}$. Let $\widetilde{\sigma}^{2}=\widetilde{\sigma} \circ \widetilde{\sigma}$. Then, $f^{\widetilde{\Delta}}(\widetilde{\sigma}(x)) / \psi(x)$ is bounded below on $\widetilde{\mathbb{T}}$ by assumptions (H3)-(H4). Indeed, in case $\tilde{\sigma}(x)=\widetilde{\sigma}^{2}(x)$, we have $f^{\prime}(\widetilde{\sigma}(x)) / \psi(x)=f^{\tilde{\Delta}}(\tilde{\sigma}(x)) / \psi(x)$ and the assertion holds. In case $\widetilde{\sigma}(x)<\widetilde{\sigma}^{2}(x)$, by the definition of delta-derivative and the mean value theorem, there exits a constant $c \in\left[\widetilde{\sigma}(x), \widetilde{\sigma}^{2}(x)\right]$ such that

$$
f^{\tilde{\Delta}}(\tilde{\sigma}(x))=\frac{f\left(\tilde{\sigma}^{2}(x)\right)-f(\tilde{\sigma}(x))}{\tilde{\sigma}^{2}(x)-\tilde{\sigma}(x)}=f^{\prime}(c),
$$

and then

$$
\frac{f^{\tilde{\Delta}}(\tilde{\sigma}(x))}{\psi(x)}=\frac{f^{\prime}(c)}{\psi(x)} \geq \frac{m_{1}}{m_{0}}
$$

Furthermore, $f^{\tilde{\Delta}}(\tilde{\sigma}(x))$ is nondecreasing on $[0, \infty) \widetilde{T}$ by virtue of $(\mathrm{H} 4)$. Indeed, for any $x, y \in[0, \infty)_{\tilde{\mathbb{T}}}$ with $x \leq y$, there are four cases to consider. In case $\tilde{\sigma}(x)=\widetilde{\sigma}^{2}(x)$ and $\widetilde{\sigma}(y)=$ $\tilde{\sigma}^{2}(y), f^{\widetilde{\Delta}}(\tilde{\sigma}(x))=f^{\prime}(\tilde{\sigma}(x)) \leq f^{\prime}(\tilde{\sigma}(y))=f^{\tilde{\Delta}}(\tilde{\sigma}(y))$. In case $\tilde{\sigma}(x)=\tilde{\sigma}^{2}(x)$ and $\widetilde{\sigma}(y)<\widetilde{\sigma}^{2}(y)$, we have $f^{\tilde{\Delta}}(\tilde{\sigma}(x))=f^{\prime}(\widetilde{\sigma}(x))$ and

$$
f^{\widetilde{\Delta}}(\tilde{\sigma}(y))=\frac{f\left(\tilde{\sigma}^{2}(y)\right)-f(\tilde{\sigma}(y))}{\tilde{\sigma}^{2}(y)-\tilde{\sigma}(y)}=f^{\prime}\left(c_{y}\right),
$$

where $c_{y} \in\left[\widetilde{\sigma}(y), \tilde{\sigma}^{2}(y)\right]$. Since $c_{y} \geq \tilde{\sigma}(x)$, it follows that $f^{\widetilde{\Delta}}(\tilde{\sigma}(x)) \leq f^{\widetilde{\Delta}}(\widetilde{\sigma}(y))$. The other cases can be shown likewise.

As a consequence, we see from assumption (H3) that $f^{\Delta}(\tilde{\sigma}(x)) / \psi(x)$ is nondecreasing on $[0, \infty)_{\widetilde{\mathbb{T}}}$. Similarly, we can show that $f^{\widetilde{\Delta}}(\widetilde{\sigma}(x)) / \psi(x)$ is nonincreasing on $(-\infty, 0]_{\tilde{\mathbb{T}}}$ provided $\tilde{\sigma}(x) \in(-\infty, 0]_{\tilde{\mathbb{T}}}$. 
As thus, we can extract the essences as above and obtain a result as follows.

Lemma 2.1. Suppose that $\tilde{\mathbb{T}}$ is a time scale and $f^{\tilde{\Delta}}(x) / \psi(x)$ is defined on it. Then it follows that

(i) $f^{\tilde{\Delta}}(\widetilde{\sigma}(x)) / \psi(x)$ is positively bounded below and

(ii) $f^{\widetilde{\Delta}}(\tilde{\sigma}(x)) / \psi(x)$ is nondecreasing on $[0, \infty)_{\tilde{\mathbb{T}}}$ and nonincreasing on $(-\infty, 0]_{\tilde{\mathbb{T}}}$.

For a given $a \in \tilde{\mathbb{T}}$ with $a \neq 0$, we introduce a function on $\widetilde{\mathbb{T}}$ as follows:

$$
\Gamma_{a}(x)=\int_{a}^{x} \frac{\psi(\tilde{\rho}(u))}{f(u)} \widetilde{\Delta} u \quad \text { for } x \in \tilde{\mathbb{T}} \text { with } x \neq 0
$$

Then the function $\Gamma_{a}(x)$ possesses the following properties.

(i) If $a>0$, then $\Gamma_{a}(x)$ is strictly increasing for $x>0$ and $\Gamma_{a}^{-1}(y) \geq a$ is strictly increasing for $y \geq 0$.

(ii) If $a<0$, then $\Gamma_{a}(x)$ is strictly decreasing for $x<0$ and $\Gamma_{a}^{-1}(y) \leq a$ is strictly decreasing for $y \geq 0$.

(iii) If $a \neq 0$, then $F\left(\Gamma_{a}^{-1}(y)\right)$ is nondecreasing for $y \geq 0$ (by Lemma 2.1 (ii)). Here $F$ is defined as in (2.6).

For the sake of convenience, we let

$$
\begin{gathered}
R(t)=\frac{p(t) \psi(x(t))\left(x^{\sigma}(t)\right)^{\Delta}}{f\left(x^{\sigma}(t)\right)}, \\
F(x)=\frac{f^{\tilde{\Delta}}(\tilde{\sigma}(x))}{\psi(x)}
\end{gathered}
$$

whenever they are defined.

Note that, if $x(t)$ is an eventually negative solution of (1.2), then $y(t)=-x(t)$ satisfies that

$$
\left(p(t)(\tilde{\psi} \circ y)\left(y^{\sigma}\right)^{\Delta}\right)^{\Delta}+q(t) \tilde{f} \circ y^{\sigma}=0
$$

where

$$
\tilde{\psi}(y)=\psi(-y), \quad \tilde{f}(y)=-f(-y)
$$

possess the same properties as $\psi$ and $f$, respectively. Therefore, in what follows we will restrict our attention to the eventually positive solutions of (1.2).

\section{Main Results}

Before entering our main discussions, we remark that $x\left(\left[t_{1}, \infty\right)_{\mathbb{T}}\right)$ is a time scale when $x \in C_{\mathrm{rd}}^{1}\left[t_{1}, \infty\right)_{\mathbb{T}}$ and $x$ is monotonic on $\left[t_{1}, \infty\right)_{\mathbb{T}}$, where $t_{1} \in \mathbb{T}$. In the following discussions, 
the notations $\tilde{\Delta}, \tilde{\sigma}$ and $\tilde{\rho}$ will act upon the time scale $x\left(\left[t_{1}, \infty\right)_{\mathbb{T}}\right)$, while $\Delta, \sigma$, and $\rho$ will do on $\mathbb{T}$. Then $\tilde{\sigma} \circ x=x \circ \sigma$ when $x$ is strictly increasing on $\left[t_{1}, \infty\right)_{\mathbb{T}}$.

Lemma 3.1. Suppose that $t_{1} \in \mathbb{T}$ and $x(t)$ is a solution of (1.2) with $x(t)>0$ on $\left[t_{1}, \infty\right)_{\mathbb{T}}$. Then it holds that

$$
R(t)=\int_{t}^{\infty} q(s) \Delta s+\int_{t}^{\infty} \frac{R(s) R(\sigma(s)) F(x(s))}{p(s)} \Delta s
$$

for all $t \in\left[t_{1}, \infty\right)_{\mathbb{T}}$ and

$$
R(t)=\int_{t}^{\infty} q(s) \Delta s+\int_{t}^{\infty} \frac{R(s) R(\sigma(s)) F\left(\Gamma_{x^{\sigma}\left(t_{1}\right)}^{-1}\left(\int_{t_{1}}^{\rho(s)}(R(u) \Delta u / p(u))\right)\right)}{p(s)} \Delta s
$$

for all $t \in\left[\sigma\left(t_{1}\right), \infty\right)_{\mathbb{T}}$.

Proof. Without loss of generality, let $p(t)>0$ and $q(t) \geq 0$ on $\left[t_{1}, \infty\right)_{\mathbb{T}}$. Then it follows from (1.2) that

$$
\left(p(t) \psi(x(t))\left(x^{\sigma}(t)\right)^{\Delta}\right)^{\Delta}=-q(t) f\left(x^{\sigma}(t)\right) \leq 0 \quad \forall t \in\left[t_{1}, \infty\right)_{\mathbb{T}} .
$$

We assert that

$$
p(t) \psi(x(t))\left(x^{\sigma}(t)\right)^{\Delta}>0 \quad \forall t \in\left[t_{1}, \infty\right)_{\mathbb{T}} .
$$

Otherwise, note from assumption (H2) that $q$ is not identically zero on $\left[t_{1}, \infty\right)_{\mathbb{T}},(3.3)$ implies that there exits a $t_{2} \in\left[t_{1}, \infty\right)_{\mathbb{T}}$ and a constant $m>0$ such that

$$
p(t) \psi(x(t))\left(x^{\sigma}(t)\right)^{\Delta} \leq-m \quad \forall t \in\left[t_{2}, \infty\right)_{\mathbb{T}} .
$$

Consequently we find that

$$
x^{\sigma}(t) \leq x^{\sigma}\left(t_{2}\right)-\frac{m}{m_{0}} \int_{t_{2}}^{t} \frac{\Delta s}{p(s)} \longrightarrow-\infty \quad \text { as } t \longrightarrow \infty,
$$

which contradicts $x(t)>0$ on $\left[t_{1}, \infty\right)_{\mathbb{T}}$. Since (3.4) holds, it is clear that

$$
\left(x^{\sigma}(t)\right)^{\Delta}>0, \quad R(t)>0 \quad \forall t \in\left[t_{1}, \infty\right)_{\mathbb{T}} .
$$

On the other hand, we see from (3.3) that $R(t)$ is nonincreasing on $\left[t_{1}, \infty\right)_{\mathbb{T}}$, which, associated with (3.7), means that $\lim _{t \rightarrow \infty} R(t)$ exists as a finite number.

Next we will show that

$$
\int_{t_{1}}^{\infty} \frac{R(s) R(\sigma(s)) F(x(s))}{p(s)} \Delta s<\infty .
$$


Note that $x^{\sigma}$ is strictly increasing due to assumption (H1) and (3.7); by (1.2), (1.5) and (1.6) and the chain rule [13, Theorem 1.93] we have

$$
\begin{aligned}
R^{\Delta}(t)= & \frac{\left[p(t) \psi(x(t))\left(x^{\sigma}(t)\right)^{\Delta}\right]^{\Delta} f\left(x^{\sigma^{2}}(t)\right)}{f\left(x^{\sigma}(t)\right) f\left(x^{\sigma^{2}}(t)\right)} \\
& -\frac{\left[p(\sigma(t)) \psi\left(x^{\sigma}(t)\right)\left(x^{\sigma^{2}}(t)\right)^{\Delta}\right] f^{\tilde{\Delta}}\left(x^{\sigma}(t)\right)\left(x^{\sigma}(t)\right)^{\Delta}}{f\left(x^{\sigma}(t)\right) f\left(x^{\sigma^{2}}(t)\right)} \\
= & -q(t)-\frac{p(\sigma(t)) \psi\left(x^{\sigma}(t)\right)\left(x^{\sigma^{2}}(t)\right)^{\Delta}}{f\left(x^{\sigma^{2}}(t)\right)} \cdot \frac{f^{\widetilde{\Delta}}\left(x^{\sigma}(t)\right)\left[p(\psi \circ x)\left(x^{\sigma}\right)^{\Delta}\right](t)}{p(t) \psi(x(t)) f\left(x^{\sigma}(t)\right)} \\
= & -q(t)-\frac{R(\sigma(t) R(t)) F(x(t))}{p(t)} .
\end{aligned}
$$

Taking $\Delta$-integral on (3.9) from $t_{1}$ to $t$, we obtain that

$$
R(t)-R\left(t_{1}\right)=-\int_{t_{1}}^{t} q(s) \Delta s-\int_{t_{1}}^{t} \frac{R(s) R(\sigma(s)) F(x(s))}{p(s)} \Delta s .
$$

Now that the limit of $R(t)$ exists as $t \rightarrow \infty$, by assumption (H2) and (3.10) we see that (3.8) holds. Note that $F(x(t))$ is positive bounded below (see Lemma 2.1(i)), (3.8) infers that $\lim _{t \rightarrow \infty} R(t)=0$. To sum up, it is easy to see that (3.10) yields (3.1).

Next we prove (3.2). Again note that $x^{\sigma}$ is strictly increasing on $\left[t_{1}, \infty\right)_{\mathbb{T}}$, by the substitution theorem [13, Theorem 1.98], it follows that

$$
\int_{t_{1}}^{t} \frac{R(t) \Delta s}{p(t)}=\int_{t_{1}}^{t} \frac{\psi(x(s))\left(x^{\sigma}(s)\right)^{\Delta} \Delta s}{f\left(x^{\sigma}(s)\right)}=\int_{x^{\sigma}\left(t_{1}\right)}^{x^{\sigma}(t)} \frac{\psi(\tilde{\rho}(u)) \tilde{\Delta} u}{f(u)},
$$

which, together with the definition of $\Gamma_{a}$, induces

$$
x(t)=\Gamma_{x^{\sigma}\left(t_{1}\right)}^{-1}\left(\int_{t_{1}}^{\rho(t)} \frac{R(s) \Delta s}{p(s)}\right) \quad \forall t \in\left[\sigma\left(t_{1}\right), \infty\right)_{\mathbb{T}},
$$

where we have used $\rho(\sigma(t))=t$ (see assumption (H1)). Now let us substitute $x(t)$ in (3.12) into (3.1) and then we obtain (3.2). The proof is complete.

Theorem 3.2. Suppose that $t_{1} \in \mathbb{T}$. Then $x(t)$ is a solution of (1.2) with $x(t)>0$ on $\left[t_{1}, \infty\right)_{\mathbb{T}}$ (or, on $\left.\left[\sigma\left(t_{1}\right), \infty\right)_{\mathbb{T}}\right)$ if and only if there exists a constant $\alpha>0$ and a function $\varphi \in C_{\mathrm{rd}}\left(\left[t_{1}, \infty\right)_{\mathbb{T}}, \mathbb{R}^{+}\right)$such that

$$
\varphi(t) \geq \int_{t}^{\infty} q(s) \Delta s+\int_{t}^{\infty} \frac{\varphi(s) \varphi(\sigma(s))}{p(s)} F\left(\Gamma_{\alpha}^{-1}\left(\int_{t_{1}}^{\rho(s)} \frac{\varphi(u) \Delta u}{p(u)}\right)\right) \Delta s
$$

for all $t \in\left[\sigma\left(t_{1}\right), \infty\right)_{\mathbb{T}}$. 
Proof. Suppose that $x(t)$ is a solution of $(1.2)$ with $x(t)>0$ on $\left[t_{1}, \infty\right)_{\mathbb{T}}$. Then, by Lemma 3.1, $R$ defined as in (2.5) satisfies $R \in C_{\mathrm{rd}}\left(\left[t_{1}, \infty\right)_{\mathbb{T}}, \mathbb{R}^{+}\right)$and (3.13) holds, where $\alpha=x^{\sigma}\left(t_{1}\right)$.

Conversely, suppose that there exists a constant $\alpha>0$ and a function $\varphi \in C_{\text {rd }}\left(\mathbb{T}, \mathbb{R}^{+}\right)$ such that (3.13) holds. Let $y_{0}(t) \equiv 0$ and define a sequence of functions $\left\{y_{n}(t)\right\}_{n=0}^{\infty}$ on $\left[\sigma\left(t_{1}\right), \infty\right)_{\mathbb{T}}$ as follows:

$$
y_{n+1}(t)=\int_{t}^{\infty} q(s) \Delta s+\int_{t}^{\infty} \frac{y_{n}(s) y_{n}(\sigma(s)) F\left(\Gamma_{\alpha}^{-1}\left(\int_{t_{1}}^{\rho(s)}\left(y_{n}(u) \Delta u / p(u)\right)\right)\right)}{p(s)} \Delta s .
$$

It is clear that $0 \leq y_{1}(t) \leq \varphi(t)$ on $\left[\sigma\left(t_{1}\right), \infty\right)_{\mathbb{T}}$. Suppose that $y_{n-1}(t) \leq y_{n}(t) \leq \varphi(t)$ on $\left[\sigma\left(t_{1}\right), \infty\right)_{\mathbb{T}}$. Then, by the monotone of $F\left(\Gamma_{a}^{-1}(y)\right)$ for $y \geq 0$, we learn that

$$
y_{n+1}(t) \geq \int_{t}^{\infty} q(s) \Delta s+\int_{t}^{\infty} \frac{y_{n-1}(s) y_{n-1}(\sigma(s)) F\left(\Gamma_{\alpha}^{-1}\left(\int_{t_{1}}^{\rho(s)}\left(y_{n-1}(u) \Delta u / p(u)\right)\right)\right)}{p(s)} \Delta s=y_{n}(t)
$$

as well as

$$
y_{n+1} \leq \varphi(t)
$$

So by the mathematical induction we obtain that

$$
0 \leq y_{n}(t) \leq y_{n+1}(t) \leq \varphi(t) \quad \forall\left[\sigma\left(t_{1}\right), \infty\right)_{\mathbb{T}},
$$

which means that there exists a function $y$ such that

$$
\lim _{n \rightarrow \infty} y_{n}(t)=y(t) \leq \varphi(t) \quad \forall t \in\left[\sigma\left(t_{1}\right), \infty\right)_{\mathbb{T}}
$$

Now by Lebesgue's domination convergence theorem on time scales [14, Chapter 5], we may deduce from (3.14) that

$$
y(t)=\int_{t}^{\infty} q(s) \Delta s+\int_{t}^{\infty} \frac{y(s) y(\sigma(s)) F\left(\Gamma_{\alpha}^{-1}\left(\int_{t_{1}}^{\rho(s)}(y(u) \Delta u / p(u))\right)\right)}{p(s)} \Delta s .
$$

Let

$$
x(t)=\Gamma_{\alpha}^{-1}\left(\int_{t_{1}}^{\rho(t)} \frac{y(s) \Delta s}{p(s)}\right) .
$$


Then $x(t) \geq \alpha$ on $\left[\sigma\left(t_{1}\right), \infty\right)_{\mathbb{T}}$ and $x^{\sigma}(t)$ is strictly increasing on $\left[t_{1}, \infty\right)_{\mathbb{T}}$. Moreover, $x\left(\left[\sigma\left(t_{1}\right), \infty\right)_{\mathbb{T}}\right)$ is another time scale with $\tilde{\sigma} \circ x=x \circ \sigma$. Therefore, by the theorem on derivative of the inverse [13, Theorem 1.97] we learn that

$$
\left(x^{\sigma}(t)\right)^{\Delta}=\frac{f\left(x^{\sigma}(t)\right) y(t)}{\psi(x(t)) p(t)} \quad \text { for } t \in\left[t_{1}, \infty\right)_{\mathbb{T}} .
$$

Now from (3.20) we have

$$
\Gamma_{\alpha}\left(x^{\sigma}(t)\right)=\int_{t_{1}}^{t} \frac{y(s) \Delta s}{p(s)} \text { for } t \in\left[t_{1}, \infty\right)_{\mathbb{T}}
$$

which implies that

$$
p(t) \psi(x(t))\left(x^{\sigma}(t)\right)^{\Delta}=y(t) f\left(x^{\sigma}(t)\right),
$$

and this results in

$$
\begin{aligned}
(p(t) & \left.\psi(x(t))\left(x^{\sigma}(t)\right)^{\Delta}\right)^{\Delta} \\
& =y^{\sigma}(t) f^{\widetilde{\Delta}}\left(x^{\sigma}(t)\right)\left(x^{\sigma}(t)\right)^{\Delta}+f\left(x^{\sigma}(t)\right) y^{\Delta}(t) \\
& =y^{\sigma}(t) f^{\widetilde{\Delta}}\left(x^{\sigma}(t)\right)\left(x^{\sigma}(t)\right)^{\Delta}+f\left(x^{\sigma}(t)\right)\left[-q(t)-\frac{y(t) y(\sigma(t)) F(x(t))}{p(t)}\right] \\
& =y^{\sigma}(t) f^{\widetilde{\Delta}}\left(x^{\sigma}(t)\right)\left(x^{\sigma}(t)\right)^{\Delta}-q(t) f\left(x^{\sigma}(t)\right)-\frac{\left[p(t) \psi(x(t))\left(x^{\sigma}(t)\right)^{\Delta}\right] y^{\sigma}(t) F(x(t))}{p(t)} \\
& =-q(t) f\left(x^{\sigma}(t)\right)
\end{aligned}
$$

where we have imposed formulas (3.19) and (3.20) for the second equal sign and (3.23) for the third, respectively.

Now we see from (3.24) that $x(t)$ defined by (3.20) is a positive solution of (1.2). The proof is complete.

In the remainder of this section, we define formally a sequence of functions $\left\{Q_{n}(t)\right\}_{n=0}^{\infty}$ as follows. Let $t_{1} \in \mathbb{T}, \alpha>0$, and

$$
\begin{gathered}
Q_{0}(t)=\int_{t}^{\infty} q(s) \Delta s \\
Q_{1}(t)=\int_{t}^{\infty} \frac{Q_{0}(s) Q_{0}(\sigma(s)) F\left(\Gamma_{\alpha}^{-1}\left(\int_{t_{1}}^{\rho(s)} Q_{0}(u) \Delta u / p(u)\right)\right)}{p(s)} \Delta s
\end{gathered}
$$


as well as

$$
\begin{aligned}
& Q_{n+1}(t) \\
& \quad=\int_{t}^{\infty} \frac{\left[Q_{0}(s)+Q_{n}(s)\right]\left[Q_{0}(\sigma(s))+Q_{n}(\sigma(s))\right] F\left(\Gamma_{\alpha}^{-1}\left(\int_{t_{1}}^{\rho(s)}\left(\left(Q_{0}(u)+Q_{n}(u)\right) / p(u) \Delta u\right)\right)\right)}{p(s)} \Delta s .
\end{aligned}
$$

Then $Q_{1}(t) \leq Q_{2}(t)$. By induction, it follows that

$$
0<Q_{n}(t) \leq Q_{n+1}(t) \text { for } n=1,2,3, \ldots
$$

If $x(t)$ is a solution of (1.2) with $x(t)>0$ on $\left[t_{1}, \infty\right)_{\mathbb{T}}$ for some $t_{1} \in \mathbb{T}$, then (3.1) holds by Lemma 3.1 and hence

$$
Q_{0}(t) \leq R(t) \quad \forall t \in\left[\sigma\left(t_{1}\right), \infty\right)_{\mathbb{T}},
$$

which, together with (3.26), results in

$$
Q_{1}(t) \leq \int_{t}^{\infty} \frac{R(s) R(\sigma(s)) F\left(\Gamma_{a}^{-1}\left(\int_{t_{1}}^{\rho(s)}(R(u) \Delta u / p(u))\right)\right)}{p(s)} \Delta s \quad \forall t \in\left[\sigma\left(t_{1}\right), \infty\right)_{\mathbb{T}} .
$$

Let us now define $R_{0}(t)$ by

$$
R_{0}(t)=\int_{t}^{\infty} \frac{R(s) R(\sigma(s)) F\left(\Gamma_{a}^{-1}\left(\int_{t_{1}}^{\rho(s)}(R(u) \Delta u / p(u))\right)\right)}{p(s)} \Delta s, \quad t \in\left[\sigma\left(t_{1}\right), \infty\right)_{\mathbb{T}} .
$$

Then, in view of (3.2) and (3.30), we have

$$
Q_{0}(t)+Q_{1}(t) \leq Q_{0}(t)+R_{0}(t)=R(t) \quad \forall t \in\left[\sigma\left(t_{1}\right), \infty\right)_{\mathbb{T}} .
$$

By the mathematical induction again we educe

$$
Q_{0}(t)+Q_{n}(t) \leq R(t) \quad \forall t \in\left[\sigma\left(t_{1}\right), \infty\right)_{\mathbb{T}}, n=1,2,3, \ldots
$$

Now we learn from (3.28) and (3.33) that $\left\{Q_{n}(t)\right\}_{0}^{\infty}$ is well defined and converges to some function $Q(t)$ when $x(t)$ is a solution of $(1.2)$ with $x(t)>0$ on $\left[t_{1}, \infty\right)_{\mathbb{T}}$.

Conversely, suppose that $\left\{Q_{n}(t)\right\}_{n=0}^{\infty}$ is well defined and

$$
\lim _{n \rightarrow \infty} Q_{n}(t)=Q(t)<\infty \quad \text { on }\left[\sigma\left(t_{1}\right), \infty\right)_{\mathbb{T}}
$$


Then, by (3.28), we have $Q_{n}(t) \leq Q(t)$ for all $n \geq 1$. Hence, by Lebesgue's domination convergence theorem on time scales, we may obtain from (3.27) that

$$
Q(t)=\int_{t}^{\infty} \frac{\left[Q_{0}(s)+Q(s)\right]\left[Q_{0}(\sigma(s))+Q(\sigma(s))\right] F\left(\Gamma_{\alpha}^{-1}\left(\int_{t_{1}}^{\rho(s)}\left(\left(Q_{0}(u)+Q(u)\right) / p(u)\right) \Delta u\right)\right)}{p(s)} \Delta s .
$$

Let $\varphi(t)=Q_{0}(t)+Q(t)$. Then, from (3.35) we see that (3.13) holds. Furthermore, by means of Theorem 3.2, (1.2) has a solution $x(t)>0$ on $\left[\sigma\left(t_{1}\right), \infty\right)_{\mathbb{T}}$.

To sum up, we obtain our last result as follows.

Corollary 3.3. Suppose that $t_{1} \in \mathbb{T}$. Then $x(t)$ is a solution of (1.2) with $x(t)>0$ on $\left[t_{1}, \infty\right)_{\mathbb{T}}$ (or, on $\left.\left[\sigma\left(t_{1}\right), \infty\right)_{\mathbb{T}}\right)$ if and only if there exists a constant $\alpha>0$ such that the sequence of functions $\left\{Q_{n}(t)\right\}_{n=0}^{\infty}$ defined as in (3.25)-(3.27) is well defined and

$$
\lim _{n \rightarrow \infty} Q_{n}(t)=Q(t)<\infty \quad \text { on }\left[\sigma\left(t_{1}\right), \infty\right)_{\mathbb{T}}
$$

Example 3.4. Let $\mathbb{T}=[0, \infty)$. Suppose in (1.2) that $p(t)=t+1, q(t)=1 /\left(4 t^{2}\right), \psi(x)=1 / 2(1+|x|)$, and $f(x)=x$. Let $\varphi(t)=1 /(2 t)$. It is easy to verify that

$$
\Gamma_{1}^{-1}\left(\int_{1}^{s} \frac{\varphi(s) \mathrm{d} s}{p(s)}\right)=s, \quad F\left(\Gamma_{1}^{-1}\left(\int_{1}^{s} \frac{\varphi(u) \mathrm{d} u}{p(u)}\right)\right)=\frac{1}{2(1+|s|)} \quad \text { for } s \geq 1
$$

as well as

$$
\int_{t}^{\infty} q(s) \mathrm{d} s+\int_{t}^{\infty} \frac{\varphi^{2}(s)}{p(s)} F\left(\Gamma_{1}^{-1}\left(\int_{1}^{s} \frac{\varphi(u) \mathrm{d} u}{p(u)}\right)\right) d s=\frac{3}{8 t} \leq \varphi(t), \quad t \geq 1
$$

By Theorem 3.2, (1.2) has a solution $x(t)>0$ on $[1, \infty)$.

Example 3.5. Let $h>0$ and $q_{0}>1$, and let $\mathbb{N}_{0}$ be a set of nonnegative integers. Suppose in (1.2) that $\psi(x)=1$ and $f(x)=x$.

In case $\mathbb{T}=h \mathbb{N}_{0}$, let

$$
p(t)=1, \quad q(t)=\frac{1}{(2 t+2 h)(2 t+h)}
$$

Then, for a given $\varphi(t)=1 /(2 t+h)$ and any given $\alpha>0$, we have

$$
\begin{gathered}
\int_{t}^{\infty} q(s) \Delta s \leq \int_{t}^{\infty} \frac{\Delta t}{\sigma(2 s+h)(2 s+h)}=\frac{1}{2(2 t+h)} \\
\int_{t}^{\infty} \frac{\varphi(s) \varphi(\sigma(s))}{p(s)} F\left(\Gamma_{\alpha}^{-1}\left(\int_{0}^{\rho(s)} \frac{\varphi(u) \Delta u}{p(u)}\right)\right) \Delta s=\int_{t}^{\infty} \frac{\Delta s}{(2 s+h)(2 \sigma(s)+h)} \leq \frac{1}{2(2 t+h)}
\end{gathered}
$$


and hence (3.13) holds. By means of Theorem 3.2, (1.2) has a solution $x(t)>0$ on $\mathbb{T}=h \mathbb{N}_{0}$. In case $\mathbb{T}=\left\{q_{0}^{k}: k \in \mathbb{N}_{0}\right\}$, let

$$
p(t)=2, \quad \varphi(t)=\frac{1}{t}, \quad q(t)=\frac{1}{2 \sigma(t) t} .
$$

Then, for any given $\alpha>0$, we have

$$
\int_{t}^{\infty} q(s) \Delta s+\int_{t}^{\infty} \frac{\varphi(s) \varphi(\sigma(s))}{p(s)} F\left(\Gamma_{\alpha}^{-1}\left(\int_{1}^{\rho(s)} \frac{\varphi(u) \Delta u}{p(u)}\right)\right) \Delta s=\varphi(t),
$$

and hence Theorem 3.2 implies that (1.2) has a solution $x(t)>0$ on $\mathbb{T}=\left\{q_{0}^{k}: k \in \mathbb{N}_{0}\right\}$.

\section{References}

[1] R. P. Agarwal, D. O'Regan, and S. H. Saker, "Oscillation criteria for nonlinear perturbed dynamic equations of second-order on time scales," Journal of Applied Mathematics E Computing, vol. 20, no. 1-2, pp. 133-147, 2006.

[2] H. A. Agwo, "Oscillation of nonlinear second order neutral delay dynamic equations on time scales," Bulletin of the Korean Mathematical Society, vol. 45, no. 2, pp. 299-312, 2008.

[3] D. R. Anderson, "Interval criteria for oscillation of nonlinear second-order dynamic equations on time scales," Nonlinear Analysis: Theory, Methods \& Applications, vol. 69, no. 12, pp. 4614-4623, 2008.

[4] D. R. Andersona and A. Zafer, "Nonlinear oscillation of second-order dynamic equations on time scales," Applied Mathematics Letters, vol. 22, no. 10, pp. 1591-1597, 2009.

[5] M. Bohner and C. C. Tisdell, "Oscillation and nonoscillation of forced second order dynamic equations," Pacific Journal of Mathematics, vol. 230, no. 1, pp. 59-71, 2007.

[6] L. Erbe, A. Peterson, and S. H. Saker, "Kamenev-type oscillation criteria for second-order linear delay dynamic equations," Dynamic Systems and Applications, vol. 15, no. 1, pp. 65-78, 2006.

[7] T. S. Hassan, "Kamenev-type oscillation criteria for second order nonlinear dynamic equations on time scales," Applied Mathematics and Computation, vol. 217, no. 12, pp. 5285-5297, 2011.

[8] J. Baoguo, L. Erbe, and A. Peterson, "Oscillation of a family of $q$-difference equations," Applied Mathematics Letters, vol. 22, no. 6, pp. 871-875, 2009.

[9] S. H. Saker, "Oscillation of nonlinear dynamic equations on time scales," Applied Mathematics and Computation, vol. 148, no. 1, pp. 81-91, 2004.

[10] S. H. Saker, "Boundedness of solutions of second-order forced nonlinear dynamic equations," The Rocky Mountain Journal of Mathematics, vol. 36, no. 6, pp. 2027-2039, 2006.

[11] S. H. Saker, "Oscillation of second-order forced nonlinear dynamic equations on time scales," Electronic Journal of Qualitative Theory of Differential Equations, no. 23, pp. 1-17, 2005.

[12] C. D. Ahlbrandt, M. Bohner, and J. Ridenhour, "Hamiltonian systems on time scales," Journal of Mathematical Analysis and Applications, vol. 250, no. 2, pp. 561-578, 2000.

[13] M. Bohner and A. Peterson, Dynamic Equations on Time Scales: An Introduction with Applications, Birkhäuser, Boston, Mass, USA, 1st edition, 2001.

[14] M. Bohner and A. Peterson, Advances in Dynamic Equations on Time Scales, Birkhäuser, Boston, Mass, USA, 2003. 


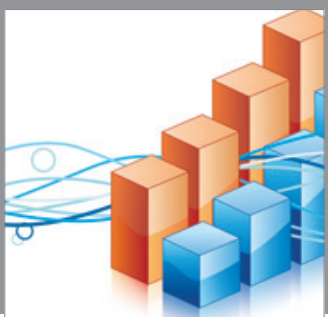

Advances in

Operations Research

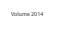

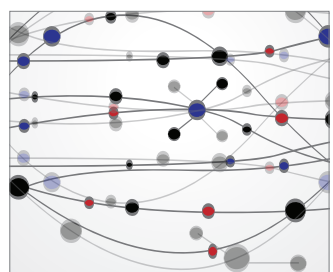

\section{The Scientific} World Journal
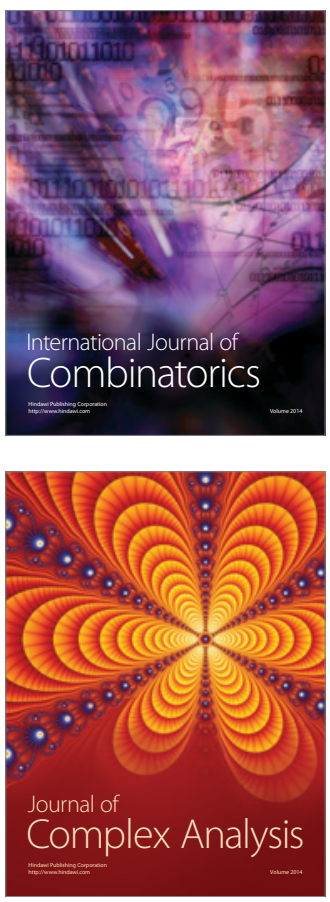

International Journal of

Mathematics and

Mathematical

Sciences
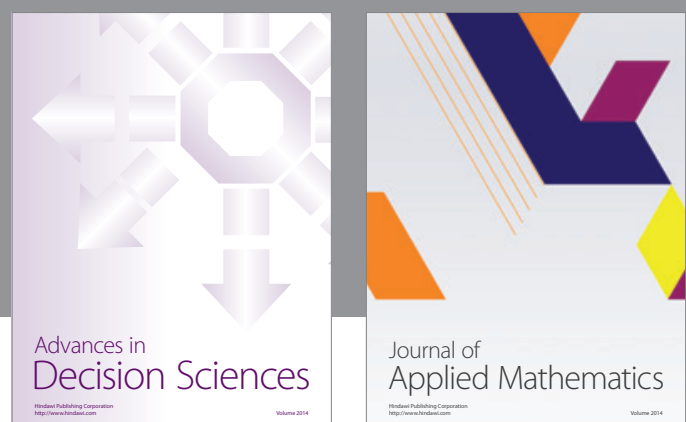

Journal of

Applied Mathematics
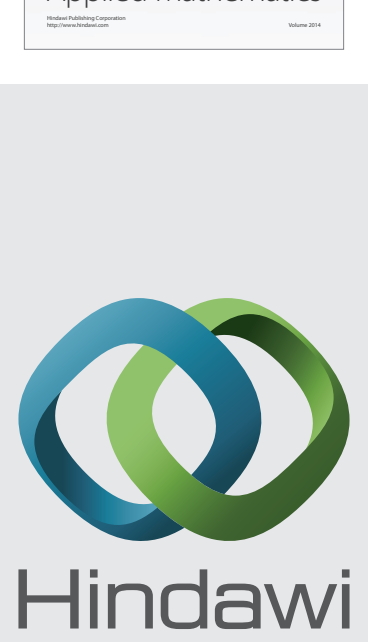

Submit your manuscripts at http://www.hindawi.com
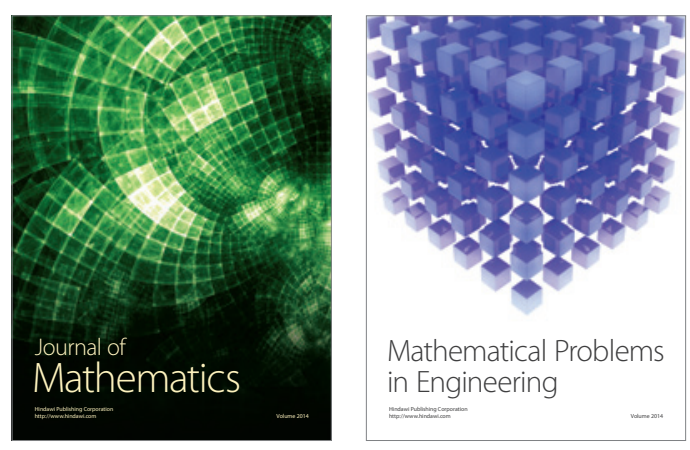

Mathematical Problems in Engineering
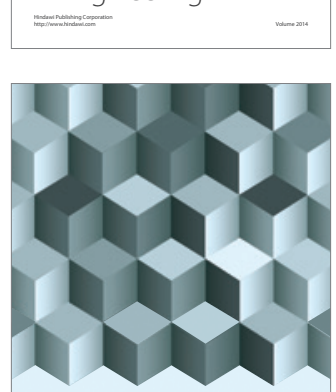

Journal of

Function Spaces
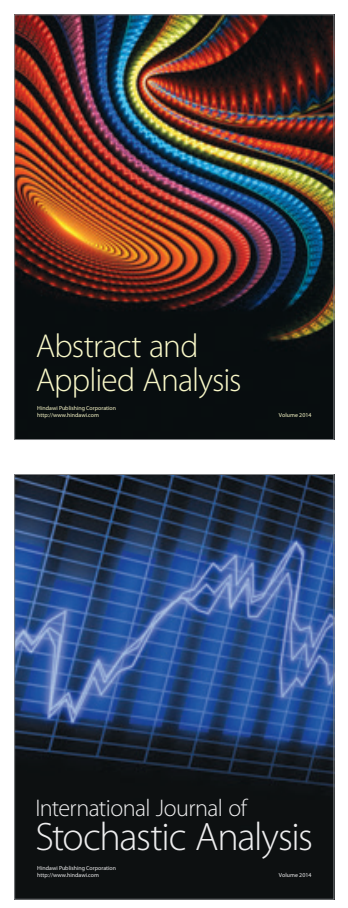

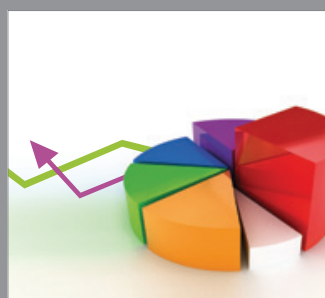

ournal of

Probability and Statistics

Promensencen
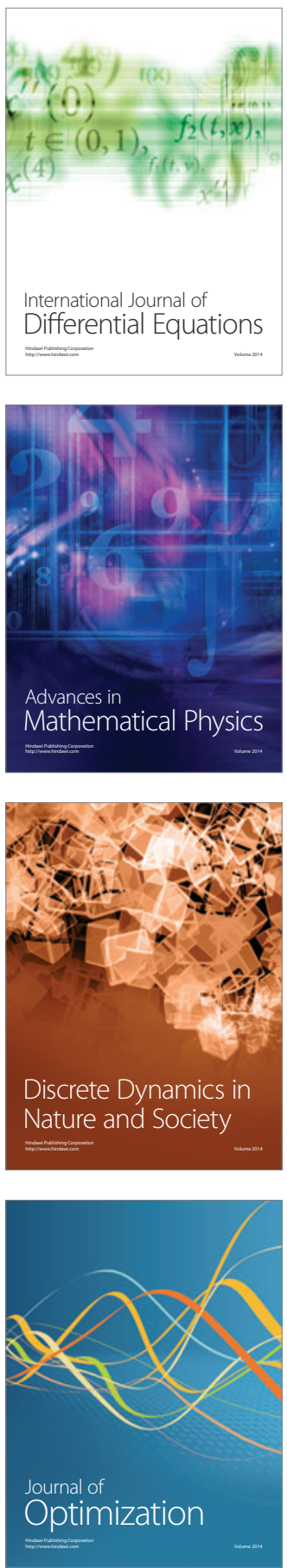